\title{
Identification of putative metabolic biomarker underlying cooked rice elongation
}

\author{
Nnaemeka Emmanuel Okpala ${ }^{1,3}$, Lixin Duan ${ }^{1}$, Guoan Shen ${ }^{1}$, Guiquan Zhang ${ }^{2}$, Xiaoquan $\mathrm{Qi}^{1}{ }^{*}$ \\ ${ }^{1}$ Key Laboratory of Plant Molecular Physiology, Institute of Botany, Chinese Academy of Sciences, Beijing \\ 100093, China \\ ${ }^{2}$ Guangdong Key Laboratory of Plant Molecular Breeding, College of Agriculture, South China Agricultural \\ University, Guangzhou 510642, China \\ ${ }^{3}$ University of Chinese Academy of Sciences, Beijing, China
}

*Corresponding author: xqi@ibcas.ac.cn

\begin{abstract}
Rice (Oryza sativa L.) is a staple food for over half of the world's population. However, rice grain quality is one of the major problems facing rice breeders across the world. Cooked rice elongation, cooked rice expansion, and water absorption have been identified as some of the parameters used in gauging rice grain quality. Biomarkers such as proteins or metabolites can be used to differentiate among a pattern of variations among various samples (e.g., various locations within a plant, various germplasm accessions); consequently, they present a type of internal validation for a given biological state. In the present study, we investigated the putative metabolite biomarkers associated with the variation of cooked rice elongation for Hua Jing Xian 74 (receptor), Basmati 370 (donor), and five hybrid lines resulting from a cross of these parent lines. We also investigated their cooked rice expansion and water absorption properties. After carrying out cooked rice elongation studies, metabolomics studies, correlation analyses, V-plot analyses, and thorough searches in public metabolite databases (Metlin, Massbank and KEGG), and in-house secondary metabolite database, we identified a metabolite with molecular weight of 280.25 and retention time of 6.4 min as a putative biomarker associated with cooked rice elongation in the varieties investigated. We also discovered that changes in cooked rice elongation and changes in cooked rice expansion follow a similar pattern; however, it appears that cooked rice elongation and cooked rice expansion do not affect water absorption in these rice lines. Our findings may facilitate the improvement of the cooked rice elongation of hybrids resulting from the crosses of Basmati 370 and Hua Jing Xian 74. Our results also offer interesting insight into cooked rice elongation, cooked rice expansion, and water absorption.
\end{abstract}

Keywords: Cooked rice elongation, cooked rice expansion, water absorption, biomarker, Basmati 370, Hua Jing Xian 74, rice grain quality.

Abbreviations: B-385_Basmati 385; HJX-74_Hua Jing Xian 74; UPLC-Q-TOF/MS_Ultra high performance liquid chromatography coupled to quadrupole time of flight mass spectrometry.

\section{Introduction}

Rice (Oryza sativa L.) is the staple food for over half of the world's population (Hu et al., 2004), and the only cereal crop that is consumed almost exclusively by human (Khush, 1997). Thousands of rice varieties have been produced through the process of the plant breeding. But there are large genetic diversities displayed among these varieties (Huang et al., 2012). These can help explain why rice is so flexible in contributing to human nutrition in many diverse locations and among consumers with varying tastes. Rice grain quality remains an important concern for rice breeders, producers, and consumers (Bagchi et al., 2015; Fan et al., 2005; Koutroubas et al., 2004). Improvement of rice grain quality is an aim of most rice breeders, as it affects the commercial value of the crop (Koutroubas et al., 2004). Cooked rice elongation, cooked rice expansion, and water absorption were identified by Tian et al., (2005) as some of the parameters used in determining rice grain quality. Many rice consumers are known to have a preference for rice grains with extensive elongation after cooking. Genetic analyses of rice grain length and width have shown that grain shape is quantitatively inherited (Chen et al., 1998). Rice grain shape has been described to be simultaneously controlled by triploid endosperm genes, cytoplasmic genes, embryo and maternal plant genes (Shi and Zhu, 1996; Shi et al., 2000). Studies by Kharabian-Masouleh et al., 2012 and Tan et al., 1999 reported that rice starch is controlled in the waxy gene region. Tian et al., 2005 reported that cooked rice elongation is also likely controlled in the waxy gene region. However, to the best of our knowledge, there have been no reports to date on any metabolic biomarkers associated with cooked rice elongation. A biomarker is a quantifiable or assessable indicator of any biological state or condition. The ability of biomarkers to faithfully represent the pattern of variation among the tissues sampled at various locations within the plant and at different plant ages provides a type of internal validation (Tarpley et al., 2005). Certain amino acids or combinations of them have been proposed as biomarkers metabolites of several metabolic processes in plants (Foyer et al., 2003). The aim of this study was to: (i) identify the putative metabolic biomarker associated with cooked rice elongation, (ii) to investigate cooked rice expansion and water absorption. 


\section{Results and Discussion}

\section{Cooked rice elongation}

The cooked rice elongation result in Fig 1 shows that the length of Basmati 370 increased by an average of $102 \%$, followed by the lengths of 198 and 199 that increased by an average of $94 \%$ and $91 \%$ respectively. On the other hand, the lengths of 197 and 226 increased by an average of $84 \%$ and $79 \%$ respectively. Hua Jing Xian 74 and 220 recorded shorter increases in length with an average of $69 \%$ and $68 \%$ respectively. Rice consumers are known to prefer grains with extensive increase in length after cooking (Tian et al., 2005).

\section{Metabolomics and statistical analyses}

A total of 7,589 ion features were identified after carrying out metabolomics data analysis according to Smith et al. (2006). 2,484 of these ion features were statistically significant at $p$ value $<0.05$. The concatenated metabolomics data were used to perform principal component analysis (PCA) and the result is shown in Fig 2. The PCA shows there were differences in the seed metabolomes of the samples. PC1 and PC2 can account for $21.8 \%$ and $12 \%$ of the observed variation, respectively.

Pearson correlation analysis was carried out using the elongation data and metabolomics data to determine metabolites that correlated with elongation. Afterwards pairwise V-plot analyses were performed on the lines to extract discriminating metabolites.

Collectively, these analyses identified a putative metabolite with a molecular weight of 280.25 and retention time of 6.4 min as a candidate metabolic biomarker associated with cooked rice elongation among the analysed samples. Fig 3 shows the peak area comparisons of the metabolites among all the lines. The linear regression model in Fig 4, with $\mathrm{R}^{2}=$ 0.67 , shows that cooked rice elongation decreased linearly along with decreasing abundance of the metabolics biomarker. Several reports (Shi and Zhu, 1996; Shi et al., 2000; Tian et al., 2005), have speculated about certain genes that control cooked rice elongation, but this is to our knowledge the first report on any metabolic biomarker associated with cooked rice elongation.

\section{Cooked rice expansion}

Cooked rice expansion is another parameter used to measure rice grain quality (Tian et al., 2005). The result of cooked rice expansion in Fig 5 shows that Basmati 370 expanded by an average of $105 \%$, followed by 198 and 199 which expanded by an average of $91 \%$ and $89 \%$ respectively; 197 and 226 expanded by an average of $85 \%$ and $81 \%$ respectively. Hua Jing Xian 74 and 220 both expanded by an average of $74 \%$ after cooking. We also found out that the expansion percentage and the elongation percentage of the rice varieties investigated in this study followed a similar trend.

\section{Water absorption percentage}

Fig 6 shows that the weight of Basmati 370 increased by an average of $291 \%$ after water absorption. While the weight of Hua Jing Xian 74 increased by an average of $201 \%$ after water absorption. The weights of 197, 198, and 226 had similar increases of $225 \%, 220 \%$, and $225 \%$, respectively, after water absorption. The weights of 199 and 220 increased by an average of $207 \%$ and $213 \%$, respectively, after water absorption. The water absorption percentage results of the rice varieties were inconsistent with the trend seen in cooked rice elongation and cooked rice expansion. The reason for the inconsistency may relate to their starch granule morphology, which has been widely reported (Singh et al., 2003; Lisle et al., 2000) to affect water absorption.

\section{Materials and Methods}

\section{Rice grains}

The five hybrid lines (see Table 1) we used in this study were crossed from Basmati 370 (donor) and Hua Jing Xian 74 (receptor). We received all rice grains from Prof. Guiquan Zhang of South China Agricultural University. Basmati 370 was chosen to be crossed with a local rice cultivar Hua Jing Xian 74, because of the uniquely long grains, soft and fluffy texture, and pleasant aroma during and after cooking. Basmati 370 is an indica rice variety (Mumm et al., 2016); Hua Jing Xian 74 is also an indica variety (Xi et al., 2006). All rice grains were cultivated in Hainan, China in November, 2013 and were harvested and stored under the same condition in April, 2014.

Table 1. List of five hybrids lines from a cross of Hua Jing Xian 74 (receptor) and Basmati 370 (donor).

\begin{tabular}{ll}
\hline Pedigree code & Metaphor code \\
\hline lxx7-2-124-12-3 & 197 \\
Lxx8-3-31-2-5-6 & 198 \\
LXX8-3-31-2-5-7 & 199 \\
Q64 & 220 \\
S38 & 226 \\
\hline
\end{tabular}

\section{Dehusking, milling and pulverizing}

Whole and mature grains were dehusked using Jing Ao Liang Yong Qi Cai Chang JLGJ-45 de-husker machine. Rice grains that got broken during the process were discarded, as were immature grains. Whole and mature dehusked rice grains were milled with a Taizhou Jing Ao LTJM-2008 milling machine for $3 \mathrm{~min}$. The objective of milling was to remove rice bran, which consists of the aleurone and pericarp tissues. Milled rice grains were pulverized with a Retsch Mixer Mill MM 400. The frequency was set at $30 / \mathrm{sec}$ and the grains were milled for $50 \mathrm{sec}$. Note that the mixer mill was extremely efficient for these samples; the samples remained cool after pulverizing.

\section{Cooked rice elongation}

Cooked rice elongation was here represented as the difference between the length of grains measured before and after cooking. In this kind of investigation, precision is very crucial; therefore a Microtek ScanMaker i56 scanner was used to make all of the length measurements.

\section{Raw rice and cooked rice measurement}

For each line, 60 grains of milled rice were measured. 20 raw grains were measured at a time in ScanMaker i56. Each grain was then transferred to a PCR plate containing $155 \mu$ of distilled water. The grains were allowed to soak for $30 \mathrm{~min}$. The PCR plate containing the rice grains was then placed in a PCR thermocycler and the rice grains were individually cooked for $20 \mathrm{~min}$ at $98^{\circ} \mathrm{C}$ and then for $5 \mathrm{~min}$ at $20^{\circ} \mathrm{C}$. The cooked rice grains were then removed from the PCR plate and placed on a filter paper. After drying for $5 \mathrm{~min}$ at room temperature, they were transferred to a petri dish to be re- 


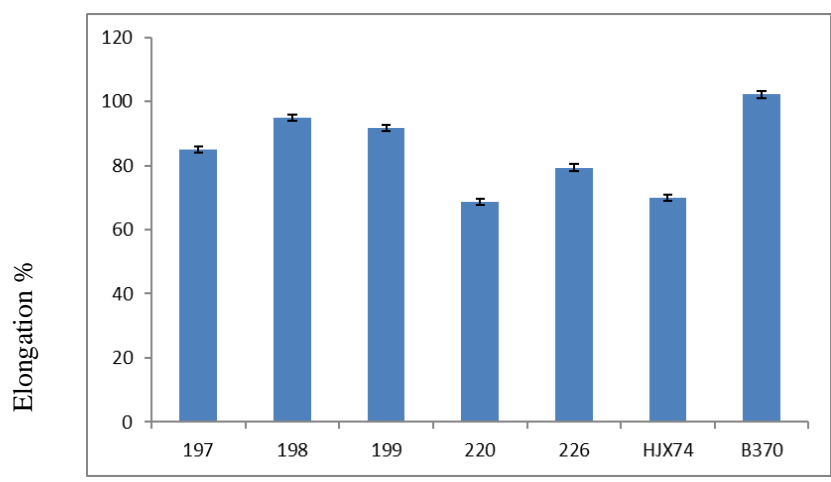

Rice lines

Fig 1. Cooked rice elongation of Hua Jing Xian 74, Basmati 370 , and five hybrid lines resulting from a cross of these parents. The codes: 197, 198, 199, 220, and 226 were explained in Table 1 .

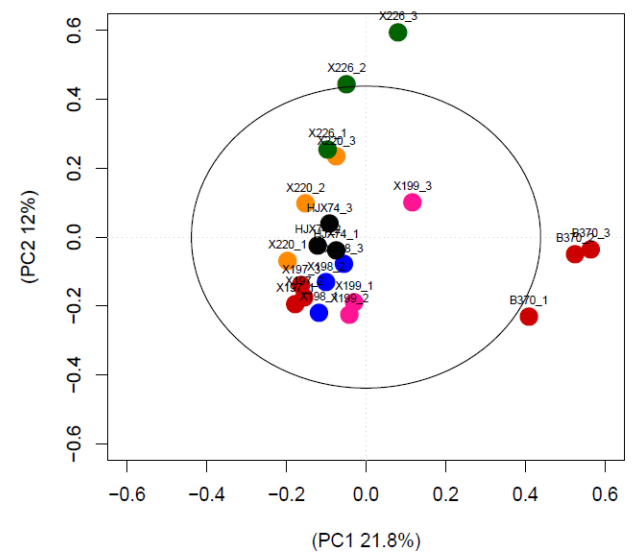

Fig 2. PCA result of the concatenated metabolomic data from different samples used in this study. A score plot of the concatenated data showing PC1 versus PC2 explaining 21.8\% and $12 \%$ variation respectively. Different coloured symbol represents different group. The codes: 197, 198, 199, 220, and 226 were explained in Table 1, while HJX 74 and B 370 were explained in abbreviations.

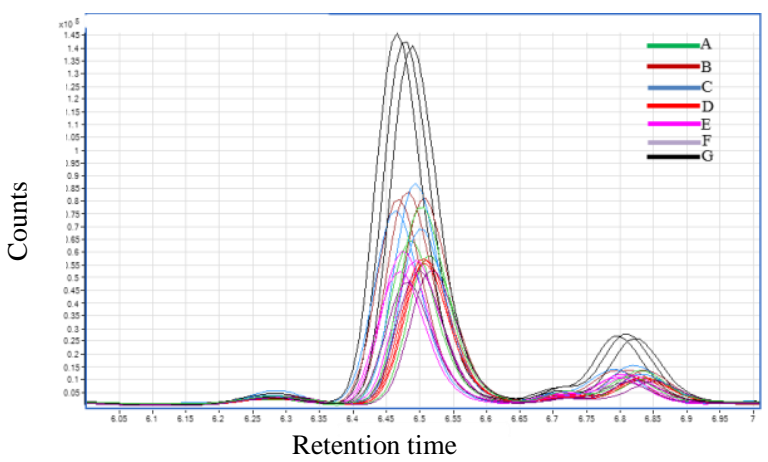

Fig 3. Metabolic biomarker abundance of the rice lines. Each line has three replicates. Different coloured symbol and letter represent different lines: A is 197; B is 198; C is 199; D is 220; $\mathrm{E}$ is 226; $\mathrm{F}$ is HJX-74; $\mathrm{G}$ is $\mathrm{B}-370$. The metabolic abundance decreased proportionately with cooked rice elongation. B-370 with the greatest extent of cooked rice elongation, also had the largest metabolic abundance. The codes: 197, 198, 199, 220, and 226 were explained in Table 1, while HJX 74 and B 370 were explained in abbreviations. measured with the scanner. As with the raw grains, 20 cooked grains were re-measured simultaneously; this helped to control the variability of water content that could have resulted from unequal resting times between measurements. These experiments used 20 grains per cultivar per each of three replicates.

\section{Cooked rice elongation}

In other to determine the elongation percentage of the cooked rice for each variety, the mean value of each replicate (before and after cooking) was determined. The following formula was used to determine the elongation percentage:

$\% \mathrm{E}=\frac{\mathrm{ACML}-\mathrm{BCML}}{\mathrm{BCML}} X 100$

where $\% \mathrm{E}$ is elongation percentage; $\mathrm{ACML}$ is the mean length after cooking and BCML is the mean length before cooking.

\section{Metabolomics analysis}

\section{UPLC-Q-TOF MS analysis}

For each cultivar, three $100 \mathrm{mg}$ samples of pulverized rice were prepared with $1,000 \mu \mathrm{l}$ of $75 \%$ methanol containing umbelliferone as an internal standard. Samples were shaken in a TNZ-C Shaker for $2 \mathrm{hr}$ at $37^{\circ} \mathrm{C}$ and $160 \mathrm{R} / \mathrm{min}$. The samples were then centrifuged for $10 \mathrm{~min}$ at $12,000 \mathrm{rpm}$. They were carefully and gently removed from the centrifuge, and $700 \mu 1$ of the sample supernatants were transferred into $1.5 \mathrm{~mL}$ glass vials with glass inserts. Blank samples consisted of $700 \mu 1$ of $75 \%$ methanol.

UPLC analysis was performed with an Agilent 1290 Infinity UHPLC system consisting of a binary pump, an autosampler, and a thermostat column compartment. The chromatography was performed using a ZORBAX RRHD SB-C18 column from Agilent Technologies $(2.1 \times 100 \mathrm{~mm}, 1.8$ $\mu \mathrm{m})$. The mobile phases were as follows; $\mathrm{H}_{2} \mathrm{O}+0.1 \%$ FA (A Phase) and Acetonitrite $+0.1 \% \mathrm{FA}$ (B Phase). The flow rate was $0.35 \mathrm{~mL} / \mathrm{min}$. The column was maintained at $40^{\circ} \mathrm{C}$. Mass spectrometry was performed using an Agilent $6540 \mathrm{Q}-\mathrm{TOF}$ instruct equipped with an electrospray ionization (ESI) source operating in positive ion mode. The nebulization gas was set at $40 \mathrm{psi}$. The drying gas was set to $11 \mathrm{~L} / \mathrm{min}$ at $325^{\circ} \mathrm{C}$ and the sheath gas was set at $11 \mathrm{~L} / \mathrm{min}$ at $325^{\circ} \mathrm{C}$. The capillary voltage was at $3500 \mathrm{~V}$.

In this study, an XCMS analysis approach was used for feature detection and calculation of chromatographic peak areas. Data analysis was performed according to Smith et al. (2006).

\section{Cooked rice expansion}

Cooked rice expansion was here represented as the difference in cooked rice grain perimeter and raw rice grain perimeter. Perimeter was used to calculate cooked rice expansion because in geometry, area is the 2-dimensional space or region occupied by a closed figure, while perimeter is the distance around a closed figure, that is, the length of the boundary. Therefore, perimeter was thought to be more appropriate.

As with cooked rice elongation, Microtek ScanMaker i56 seed scanner was used to measure the perimeter of rice seeds. However, the experimental method was slightly different. The following procedures were used for the measurements: For each replicate, 20 grains of milled rice were selected for perimeter measurement per cultivar per each of the three 


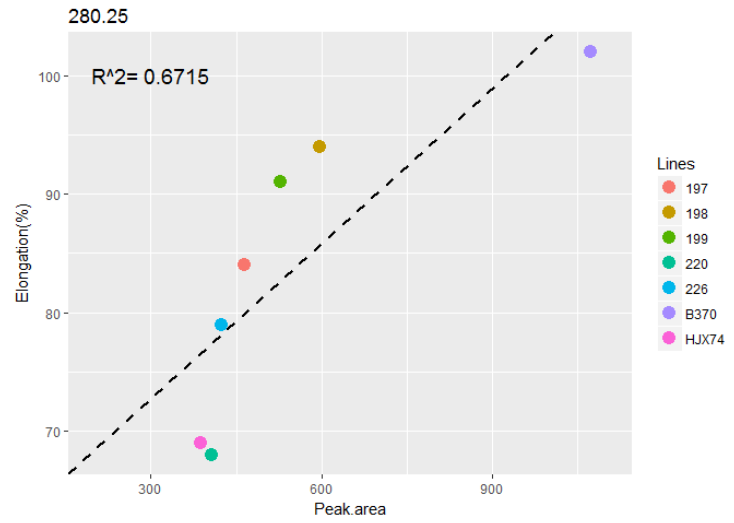

Fig 4. Linear regression model of the putative metabolic biomarker and cooked rice elongation. The linear model with $\mathrm{R}^{2}=0.67$ shows that cooked rice elongation decreased linearly along with decreasing abundance of metabolic biomarker. The coloured symbols represent different lines. The codes: 197, 198, 199, 220, and 226 were explained in Table 1, while HJX 74 and B 370 were explained in abbreviations.

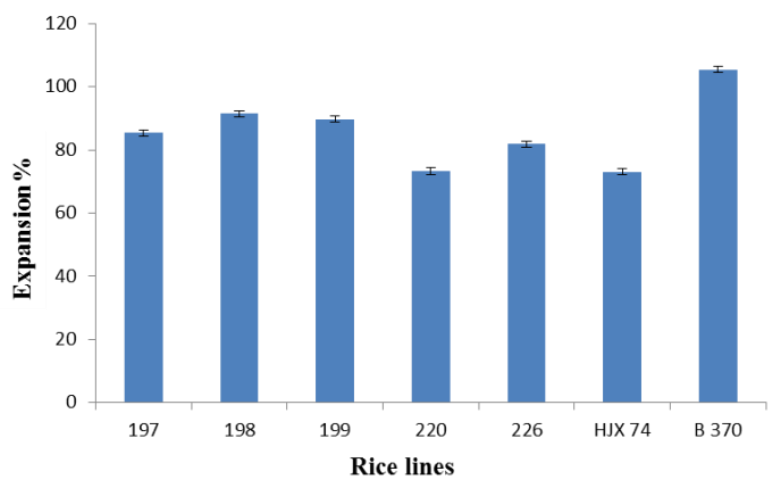

Fig 5. Cooked rice expansion of Hua Jing Xian 74, Basmati 370 , and five hybrid lines resulting from a cross of these parents. The codes: 197, 198, 199, 220, and 226 were explained in Table 1 .

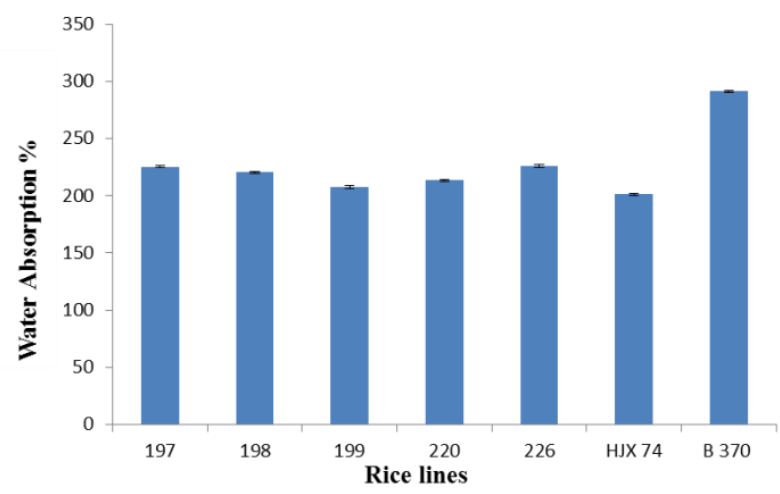

Fig 6. Water absorption of Hua Jing Xian 74, Basmati 370, and five hybrid lines resulting from a cross of these parents. The codes: 197, 198, 199, 220, and 226 were explained in Table 1 . replicates. 20 seeds were transferred in Eppendorf tube containing $1200 \mu \mathrm{l}$ of distilled water and then allowed to soak for $30 \mathrm{mins}$. The soaked grains were cooked in a water bath for $22 \mathrm{~min}$ at $98^{\circ} \mathrm{C}$. The cooked rice were then removed from the PCR plate and placed on a filter paper to dry for $5 \mathrm{~min}$ at room temperature. They were transferred to a petri dish to be re-measured in Microtek ScanMaker i56.

\section{Cooked rice expansion percentage}

In other to determine the cooked rice expansion percentage, the mean values of the three replicates before and after cooking were determined. The following formula was used:

$\%$ EXP $=\frac{\text { ACMP-BCMP }}{\text { BCMP }} X 100$

where \%Exp is expansion percentage; BCMP is before cooking mean perimeter; and ACMP is after cooking mean perimeter.

\section{Water absorption}

Water absorption in the present study, refers to the difference in weight of rice grains before cooking and after cooking. The following procedures were used to determine water absorption of cooked rice: 20 milled rice grains were selected from each cultivar (and for each cultivar three replicates were used) and were weighed in weighing machine. After weighing, the grains were transferred to Eppendorf tube and soaked in $1,000 \mu \mathrm{l}$ distilled water for $30 \mathrm{~min}$. They were cooked in a dry bath at $98^{\circ} \mathrm{C}$ for $22 \mathrm{~min}$ and then placed on a filter paper to dry for $4 \mathrm{~min}$ before being re-weighed.

\section{Water absorption percentage}

In other to determine the water absorption percentage the mean value of each replicate before and cooking were calculated. The following formula was used for water absorption percentage:

$\% \mathrm{WA}=\frac{\text { ACMW }-\mathrm{BCMW}}{\mathrm{BCMW}} X 100$

Where; \%WA is water absorption percentage; BCMW is before cooking mean weight and ACMW is after cooking mean weight.

\section{Statistics}

Principle component analysis was performed with the princomp function of pysch package in $\mathrm{R}$ 3.2.2 software ( $\mathrm{R}$ Core Team, 2015). To assess statistical difference between the rice metabolomes, Student's t-tests were used with $\mathrm{P}$ value set of $<0.05$ as the criterion of significance.

\section{Conclusion}

In summary, given that rice consumers are typically thought to desire rice grains with extensive cooked rice elongation, the findings reported in this work will be helpful in improving the cooked rice elongation resulting from the cross of Basmati 370 and Hua Jing Xian 74. More studies should also be done to see if the role of the putative biomarker is applicable in other rice varieties. This study also shows that changes in cooked rice elongation and changes in cooked rice expansion follow a similar pattern, however, it seems that cooked rice elongation and cooked rice expansion did not affect water absorption of the rice varieties. 


\section{References}

Bagchi TB, Sharma S, Chattopadhyay K (2015) Development of NIRS models to predict protein and amylose content of brown rice and proximate compositions of rice bran. Food Chem. 191:21-27.

Chen JG, Song GQ, Qu SH, Zhou Y (1998) Direct and maternal genetic effects on grain quality characters in early hybrid rice. Chin J Rice Sci. 12:79-84.

Fan CC, Yu XQ, Xing YZ, Xu CG, Luo LJ, Zhang Q (2005) The main effects, epistatic effects and environmental interactions of QTLs on the cooking and eating quality of rice in a doubled haploid line population. Theor Appl Genet. 110:1445 1452.

Foyer CH, Parry M, Noctor G (2003) Markers and signals associated with nitrogen assimilation in higher plants. J Exp Bot. 54:585-593.

Hu C, Shi J, Quan S, Cui B, Kleessen S, Nikoloski Z, Tohge T, Alexander D, Guo L, Lin H, Wang J, Cui X, Rao J, Luo Q, Zhao X, Fernie A, Zhang D (2014) Metabolic variation between japonica and indica rice cultivars as revealed by non-targeted metabolomics. Sci Rep. 4:5067.

Huang X (2012) A map of rice genome variation reveals the origin of cultivated rice. Nature. 490:497-501.

Kharabian-Masouleh A, Waters DL, Reinke RF, Ward R, Henry RJ (2012) SNP in starch biosynthesis genes associated with nutritional and functional properties of rice. Sci Rep. 2:557

Khush GS (1997) Origin, dispersal, cultivation and variation of rice. Plant Mol Biol. 35:25-34.

Koutroubas SD, Mazzini F, Pons B, Ntanos DA (2004) Grain quality variation and relationships with morphophysiological traits in rice (Oryza sativa L.) genetic resources in Europe. Field Crop Res. 86:115-130.

Lisle AJ, Martin M, Fitzgerald MA (2000) Chalky and translucent rice grains differ in starch composition and structure and cooking properties. Cereal Chem. 77:627-632.

Mumm R, Hageman JA, Calingacion MN, R. de Vos RCH, Jonker HH, Erban A, Kopka J, Hansen TH, Laursen KH, Schjoerring JK, Ward JL, Beale ML, Jongee S, Rauf A, Habibi F, Indrasari SD, Sakhan S, Ramli A, Romero M,
Reinke RF, Ohtsubo K, Boualaphanh C, Fitzgerald MA, Hall RD (2016) Multi-platform metabolomics analyses of a broad collection of fragrant and non-fragrant rice varieties reveals the high complexity of grain quality characteristics. Metabolomics. 12:38.

R Core Team (2015) R: A language and environment for statistical computing. $\mathrm{R}$ Foundation for Statistical Computing. https://www.R-project.org/.

Singh N, Singh J, Kaur L, Sodhi NS, Gill BS (2003) Morphological, thermal and rheological properties of starches from different botanical sources. Food Chem. 81:219-231.

Shi CH, Zhu J (1996) Genetic analysis of endosperm, cytoplasmic and maternal effects for exterior quality traits in Indica rice. Chin J Biomath. 11:73-81.

Shi C, Zhu J, Wu J, Fan L (2000) Genetic and genotype environment interaction effects from embryo, endosperm, cytoplasm and maternal plant for rice grain shape traits of Indica rice. Field Crops Res. 68:191-198.

Smith CA, Want EJ, O'Maille G, Abagyan R, Siuzdak G (2006) XCMS: processing mass spectrometry data for metabolite profiling using nonlinear peak alignment, matching, and identification. Anal Chem. 78(3):779-787.

Tan YF, Li JX, Yu SB, Xing YZ, Xu GC, Zhang Q (1999) The three important traits for cooking and eating quality of rice grains are controlled by a single locus in an elite hybrid, Shanyou 63. Theor Appl Genet. 99:642-648.

Tarpley L, Duran A L, Kebrom TH, Sumner LW (2005) Biomarker metabolites capturing the metabolite variance present in a rice plant developmental period. BMC Plant Biology. 5:8.

Tian R, Jiang GH, Shen LH, Wang LQ, He YQ (2005) Mapping quantitative trait loci underlying the cooking and eating quality of rice using a DH population. Mol Breeding. 15:117-124.

Xi ZY, He FH, Zeng RZ, Zhang ZM, Ding XH, Li WT, Zhang GQ (2006) Development of a wide population of chromosome single-segment substitution lines in the genetic background of an elite cultivar of rice (Oryza sativa L.). Genome. 49(5):476-84. 